$01.1 ; 03$

\title{
Тепловое состояние пакета охлаждаемых микроракетных газодинамических лазеров
}

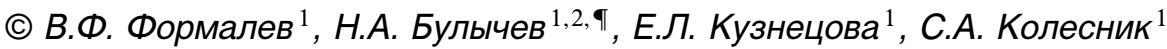 \\ ${ }^{1}$ Московский авиационный институт (Национальный исследовательский университет), Москва, Россия \\ ${ }^{2}$ Физический институт им. П.Н. Лебедева РАН, Москва, Россия \\ ๑E-mail: nbulychev@mail.ru
}

Поступило в Редакцию 5 августа 2019г.

В окончательной редакции 12 декабря 2019 г.

Принято к публикации 12 декабря 2019г.

\begin{abstract}
Приведена комплексная физико-математическая модель теплопереноса в пакетах плоских охлаждаемых микроракетных газодинамических сопел, используемых для накачки газодинамических лазеров. Особенностью таких ракетных сопел является их малый размер $(\sim 15 \mathrm{~mm})$, поэтому они быстро прогреваются, а потому необходим интенсивный теплоотвод. Комплексная физико-математическая модель теплогазодинамики, прогрева и охлаждения является новой, поскольку все физические процессы сопряжены на границе многосвязной области. Решение задачи охлаждения высокотемпературных газодинамических лазеров является одной из основных проблем при их проектировании. Получены результаты численного решения для температур газа, коэффициента теплоотдачи, температур охладителя, а также температур в критическом сечении сопла.
\end{abstract}

Ключевые слова: теплоперенос, физико-математическая модель, сопло, газодинамический лазер.

DOI: 10.21883/PJTF.2020.05.49109.18009

Базовая конструкция газодинамического лазера представляет собой сопло, через которое со сверхзвуковой скоростью (до $4 \mathrm{M}$ ) выходит перегретый до $1500^{\circ} \mathrm{C}$ газ. Мгновенное расширение и адиабатическое охлаждение газа оставляют в нем значительное количество молекул в возбужденном состоянии. Далее рабочее тело попадает в конструкцию, аналогичную газовым лазерам, где возбужденные молекулы переходят в основное состояние, участвуя в вынужденном излучении. Зачастую конструкция такого лазера основана на использовании в качестве сопел авиационных турбореактивных двигателей или ракетных двигателей. Газодинамический принцип накачки, несмотря на невысокий КПД, может давать лазерное излучение сверхвысоких энергий (до мегаватт) как в импульсном, так и в непрерывном режимах.

Рассмотрение теплового состояния охлаждаемого блока из нескольких ракетных микродвигателей (длина камеры сгорания и сопла не более $15 \mathrm{~mm}$ ) прямоугольного сечения с организованными в теле блока поперечными каналами для прохождения охладителя, в качестве которого может быть использован воздух, является важной задачей, так как такие пакеты сопел используются для накачки мощных лазеров. Источником тепловой энергии является высокотемпературное газодинамическое течение в каналах микроракетных двигателей (температура торможения $\sim 2000 \mathrm{~K}$ ).

Для определения теплового состояния необходимо решить следующий комплекс задач сопряженного теплообмена между газо- и гидродинамическим течениями и корпусом блока охлаждаемых газодинамических микродвигателей: о течении и теплообмене в теплогазодинамическом пограничном слое; о сопряженном теплообмене между газодинамическим пограничным слоем и корпусом блока двигателей; о течении в каналах охлаждения и сопряженном теплообмене между корпусом и охладителем; о нестационарном двумерном теплопереносе в блоке газодинамических микродвигателей.

По отдельным компонентам имеется значительное число публикаций, среди которых следует отметить [1-15]. Но в настоящей работе проблема сопряженного теплообмена между гидро- и газодинамическими течениями и телами сложной геометрической формы впервые решена в комплексной постановке.

На рис. 1 представлено сечение одного микродвигателя плоскостью $x O y$ в декартовой системе координат, причем продольной осью является ось $O x$, вертикальной - ось $O y$, поперечной - ось $O z$ с началом координат в начале камеры сгорания. Оси охлаждающих

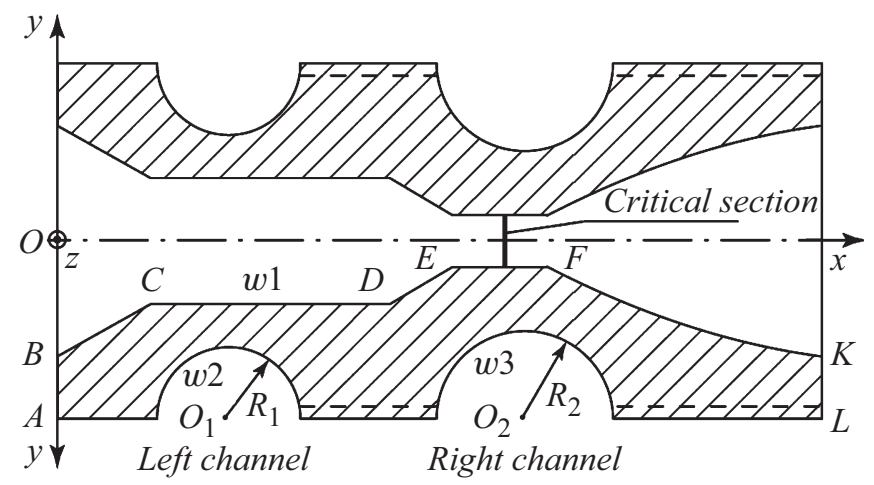

Рис. 1. Сечение отдельного ракетного микродвигателя плоскостью $x O y$ в блоке с охлаждающими каналами. 
каналов параллельны оси $O z$, причем охладитель входит в левый канал с радиусом $R_{1}$ и осью, проходящей через точку $O_{1}$, и после выхода из него входит с противоположной стороны в правый канал с радиусом $R_{2}$ и осью, проходящей через точку $\mathrm{O}_{2}$.

Физико-математическая модель комплексной проблемы содержит следующие частные математические модели, каждая из которых имеет самостоятельное значение.

1. Уравнения квазистационарного (стационарного в каждый момент времени) теплогазодинамического пограничного слоя для определения компонент вектора скорости $u(x, y), v(x, y)$, плотности $\rho(x, y)$ и температуры $T(x, y)$

$$
\begin{gathered}
\rho u \frac{\partial u}{\partial x}+\rho v \frac{\partial u}{\partial y}=-\frac{\partial p}{\partial x}+\frac{\partial}{\partial y}\left(\mu \frac{\partial u}{\partial y}\right) \\
\frac{\partial(\rho u)}{\partial x}+\frac{\partial(\rho v)}{\partial y}=0 \\
\rho u c_{p} \frac{\partial T}{\partial x}+\rho v c_{p} \frac{\partial T}{\partial y}=\frac{\partial}{\partial y}\left(\lambda \frac{\partial T}{\partial y}\right)+u \frac{d p}{d x}+\mu\left(\frac{\partial u}{\partial y}\right)^{2} \\
p=\rho R T
\end{gathered}
$$

с граничными условиями (температура и давление торможения $T_{0}, p_{0}$ в камере сгорания заданы)

$$
\begin{gathered}
y=0: \quad p_{e}(x)=p_{0}-\frac{\rho_{e}(x) u_{e}^{2}(x)}{2}, \\
T_{e}(x)=T_{0}\left(1-r \frac{k-1}{2} M_{e}^{2}\right), \quad \rho_{e}=\frac{p_{e}}{R T_{e}(x)}, \\
\left.\frac{\partial u_{e}(x)}{\partial y}\right|_{y=0}=0, \\
y=y_{w 1}: \quad u\left(x, y_{w 1}\right)=0, \quad v\left(x, y_{w 1}\right)=0, \\
p_{w 1}(x)=p_{e}(x), \quad \rho_{w 1}(x)=\frac{p_{e}(x)}{R T_{w 1}(x)}, \\
T\left(x, y_{w 1}\right)=T_{w 1},\left.\quad \lambda_{e} \frac{\partial T}{\partial n}\right|_{y=y_{w 1}+0}=\left.\lambda \frac{\partial T}{\partial n}\right|_{y=y_{w 1}-0} .
\end{gathered}
$$

Здесь $\rho-$ плотность, $\mu-$ динамическая вязкость, $\lambda-$ теплопроводность газа, $c_{p}$ - теплоемкость при постоянном давлении, $R$ - газовая постоянная, $r=0.88$, $k=c_{p} / c_{v}, c_{v}$ - теплоемкость при постоянном объеме; индексы: $e-$ на оси $O x, 0-$ торможение, $w 1-$ граница.

2. Квазистационарное течение и теплообмен охладителя в каналах в направлении оси $O z$ для ламинарного потока описываются уравнениями

$$
\begin{gathered}
\frac{\partial}{\partial r}\left(r \mu \frac{\partial u}{\partial r}\right)=r \frac{\partial p}{\partial z},\left.\quad \frac{\partial u}{\partial r}\right|_{r=0}=0,\left.\quad u\right|_{r=R_{1,2}}=0 \\
\rho v c_{p} \frac{\partial T}{\partial r}+\rho u c_{p} \frac{\partial T}{\partial z}=\frac{\lambda}{r} \frac{\partial}{\partial r}\left(r \frac{\partial T}{\partial r}\right)+\lambda \frac{\partial^{2} T}{\partial z^{2}} \\
\left.T\right|_{r=R_{s}}=T_{w s},\left.\quad T\right|_{z=0}=T_{i n c}, \quad s=1,2
\end{gathered}
$$

$$
\begin{gathered}
\left.\lambda \frac{\partial T}{\partial r}\right|_{r=R_{s}-0}-\left.\lambda \frac{\partial T}{\partial r}\right|_{r=R_{s}+0}=0, \\
\left.T\right|_{r=R_{s}-0}=T_{r=R_{s}+0}=T_{w s}, \quad s=1,2 .
\end{gathered}
$$

3. Задача нестационарной двумерной теплопроводности в многосвязном корпусе плоского сопла для определения $T(x, y, t)$ :

$$
\begin{gathered}
c(T) \rho \frac{\partial T}{\partial t}=\frac{\partial}{\partial x}\left(\lambda(T) \frac{\partial T}{\partial x}\right)+\frac{\partial}{\partial y}\left(\lambda(T) \frac{\partial T}{\partial y}\right), \\
\{x, y\} \in V, \quad t>0, \\
\left.\lambda(T) \frac{\partial T}{\partial n}\right|_{w 1}=\alpha_{w 1}\left(T_{e}-T_{w 1}\right)-\varepsilon_{w 1} \sigma T_{w 1}^{4}+\varepsilon_{e} \sigma T_{e}^{4}, \\
\{x, y\} \in S, \\
\left.\lambda(T) \frac{\partial T}{\partial r}\right|_{w s}=\alpha_{w s}\left(T_{w s}-T_{e s}\right), \quad s=1,2, \\
T(x, y, 0)=\varphi(x, y), \quad\{x, y\} \in V+S .
\end{gathered}
$$

Здесь $\alpha-$ коэффициент теплоотдачи, $\varepsilon-$ степень черноты, $\sigma$ - постоянная Стефана-Больцмана $S$ граница $B C D E F K$.

Параметры теплообмена $\alpha_{w 1}, T_{e}$ определяются исходя из системы уравнений $(1)-(6)$ по методу эффективной длины $x_{\text {eff }}[7]$ :

$$
\begin{gathered}
\alpha_{w 1}=0.0296 \operatorname{Pr}^{-0.57} K_{\mathrm{T}} \frac{\rho_{w 1} u_{e 1} \mu_{w 1}^{0.2}}{x_{e f f}^{0.2}} \frac{c_{p}}{\operatorname{Pr}}, \\
T_{e 1}=T_{0} \frac{1+r \omega}{1+\omega}, \omega=\frac{k-1}{2} \mathrm{M}_{e}^{2}, \\
K_{T}=\left(T_{w 1} / T_{e 1}\right)^{0.4+0.2 \exp (-r \omega)}(1+r \omega)^{0.11}, \\
\int_{e f f}^{x} \rho_{w 1} u_{e 1} R^{5 / 4} d x \\
\rho_{0}+\frac{x_{0}}{\rho_{w 1} u_{e 1} R^{5 / 4}}, R_{0}=\left.R\right|_{x=0} .
\end{gathered}
$$

Здесь $\operatorname{Pr}=\frac{\mu c_{p}}{\lambda}-$ число Прандля, $R_{0}-$ расстояние $B O$.

Динамические и тепловые характеристики в каналах охлаждения определяются из уравнений (7)-(9) с использованием формул из работы [8]:

$$
\begin{gathered}
u(r)=2 u_{c p}\left[1-\left(r / R_{s}\right)^{2}\right], \\
u_{c p}=\frac{1}{\pi R_{s}^{2}} \int_{0}^{R_{s}} 2 \pi r u(r) d r=-\frac{R_{s}^{2}}{8 \mu} \frac{d p}{d z}, \quad s=1,2, \\
-\frac{d p}{d z}=\frac{64}{\operatorname{Re}_{c p}} \frac{\rho u_{c p}^{2}}{2 D_{s}}, \quad \operatorname{Re}_{c p}=\frac{\dot{m} D_{s}}{F_{s} \mu}, \quad s=1,2, \\
p(z)=p_{0}-\frac{64}{\operatorname{Re}_{c p}} \frac{\left(\rho u_{c p}\right)_{0}^{2}}{2 D_{s}}, \\
\alpha_{w s}=\left\{\begin{array}{c}
\frac{3.66 \lambda}{D_{s}} \frac{\operatorname{Pe} D_{s}}{z} \leqslant 12, \\
1.61 \frac{\lambda \mathrm{Pe}}{z} \frac{\operatorname{Pe} D_{s}}{z}>12, s=1,2,
\end{array}\right.
\end{gathered}
$$




$$
\begin{gathered}
T_{c p s}(z)=\frac{2}{u_{c p} R_{s}^{2}} \int_{0}^{R_{s}} u(r) T(z) r d r, \\
\frac{T_{w s}-T(z)}{T_{w s}-T_{i n c}}=0.819 \exp (-14.62 Z) \\
+0.0189 \exp (-212 Z)+\ldots, Z=a z /\left(u_{c p} D_{s}^{2}\right) .
\end{gathered}
$$

Здесь $\operatorname{Re}=\frac{\rho u z}{\mu}, \operatorname{Pe}=\frac{u D c \rho}{\lambda}-$ числа Рейнольдса и Пекле, $D$ - диаметр каналов, $\mu, \rho, \lambda$ определяются по формулам Сезерленда $[1,7]$.

На рис. 2 и 3 приведены некоторые результаты численного решения комплексной проблемы о тепловом состоянии корпуса микродвигателя.

Входные данные принимали следующие значения: длина $l_{x}=A L=0.14 \mathrm{~m}$ (рис. 1 ), критическое сечение на длине $E F \quad 0.004 \mathrm{~m}$, расстояние на срезе сопла $0.05 \mathrm{~m}$, время работы $t \leqslant 10 \mathrm{~s}$, диаметры первого и второго каналов охлаждения равны соответственно 0.03 и $0.035 \mathrm{~mm}$, расход охладителя - воздуха - через каждый канал $\rho u=\dot{m}=131 \mathrm{~kg} /\left(\mathrm{m}^{2} \cdot \mathrm{s}\right), \lambda_{\text {air }}=0.041 \mathrm{~W} /(\mathrm{m} \cdot \mathrm{K})$, горючеe $-\mathrm{H}_{2}, R_{\mathrm{H}_{2}}=4125 \mathrm{~J} /(\mathrm{kg} \cdot \mathrm{K}), c_{p}=14270 \mathrm{~J} /(\mathrm{kg} \cdot \mathrm{K})$, $c_{v}=10130 \mathrm{~J} /(\mathrm{kg} \cdot \mathrm{K}), \quad \mu_{0}=0.88 \cdot 10^{-5} \mathrm{~kg} /(\mathrm{m} \cdot \mathrm{s})$, $\rho_{\mathrm{H}_{2}}=0.09 \mathrm{~kg} / \mathrm{m}^{3}$, скорость звука в газе $a=1286 \mathrm{~m} / \mathrm{s}$, $T_{0}=2000 \mathrm{~K}$, начальное распределение температуры во всей системе равно $\varphi(x, y)=300 \mathrm{~K}=$ const.

На рис. 2 приведены параметры теплообмена $\alpha_{w 1}, T_{e}$ на границе, омываемой высокотемпературным газодинамическим потоком. Видно резкое падение теплового потока $\alpha_{w 1}\left(T_{e 1}-T_{w 1}\right)$ в сверхзвуковой части сопла за счет значительного падения давления и плотности газа.

Рис. 3, a демонстрирует величину нагрева газообразного охладителя при течении его в левом канале в прямом направлении, а затем при течении того же охладителя в обратном направлении в правом канале. За время, равное $5.6 \mathrm{~s}$, охладитель в обоих каналах нагревается до температуры $800 \mathrm{~K}$, т.е. охладитель снимает значительное количество тепловой энергии, поступающей в корпус от газодинамического потока и нагретого корпуса.

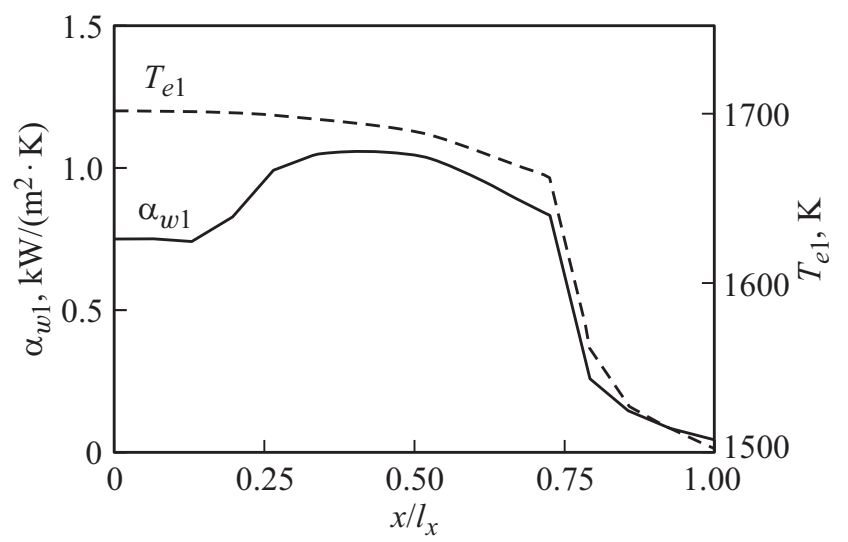

Рис. 2. Распределение температуры $T_{e 1}$ и коэффициента теплообмена $\alpha_{w 1}$ на границе микродвигателя $w 1$ в момент времени $t=2.6 \mathrm{~s}$.
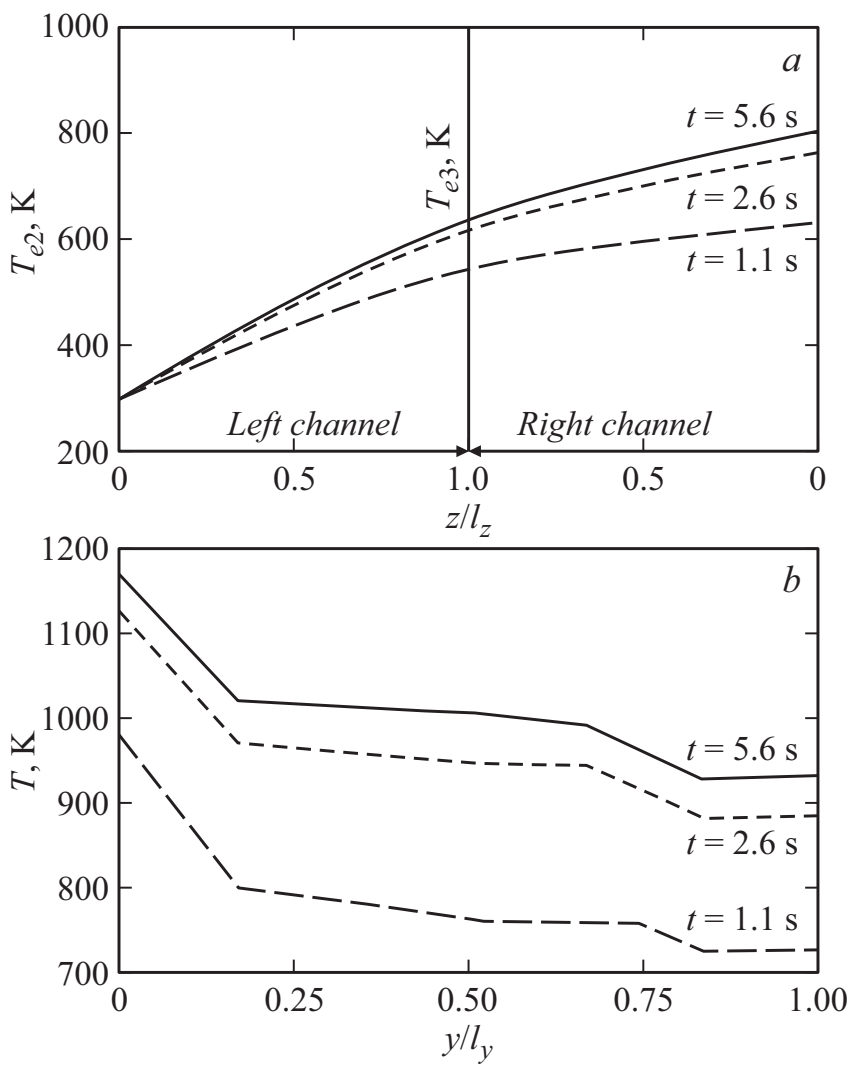

Рис. 3. $a-$ температура охладителя в левом канале при его течении в прямом направлении и в правом канале при течении в обратном направлении; $b-$ распределение температуры в критическом сечении корпуса микродвигателя.

На рис. 3, $b$ приведены результаты расчетов распределения температур в критическом сечении микроракетного двигателя, являющегося наиболее теплонапряженным. Максимальная температура на границе $w 1$ в критическом сечении, достигаемая за $5.6 \mathrm{~s}$, равна $1123 \mathrm{~K}$, что еще может выдержать стальной корпус, правда при резком снижении прочности. Для существенного снижения температуры в критическом сечении необходимо значительно увеличить расход охладителя.

Таким образом, в работе впервые поставлена комплексная задача о тепловом состоянии пакета микроракетных газодинамических сопел, используемых для накачки газодинамических лазеров. Разработан алгоритм численного решения поставленной задачи по определению параметров теплообмена на свободных границах многосвязной области. Полученные результаты подтвердили адекватность физико-математического моделирования сопряженного теплопереноса между газодинамическими течениями и многосвязными телами.

\section{Финансирование работы}

Работа выполнена при поддержке Российского фонда фундаментальных исследований (гранты 18-01-00444 и 18-01-00446). 


\section{Конфликт интересов}

Авторы заявляют, что у них нет конфликта интересов.

\section{Список литературы}

[1] Шлихтинг Г. Теория пограничного слоя. М.: Наука, 1969. $742 \mathrm{c}$.

[2] Формалев В.Ф., Колесник С.A. // Теплофизика высоких температур. 2007. Т. 45. № 1. С. 85-93.

[3] Формалев В.Ф., Колесник С.А., Кузнецова Е.Л. // Теплофизика высоких температур. 2009. Т. 47. № 2. С. 247-253.

[4] Михеев М.А., Михеева И.М. Основы теплопередачи. М.: Энергия, 1973. $320 \mathrm{c}$.

[5] Галищейский Б.М., Совершенный В.Д., Формалев В.Ф. Тепловая защита лопаток турбин. М.: Изд-во МАИ, 1996. $356 \mathrm{c}$.

[6] Основы теплопередачи в авиационной и ракетнокосмической технике / Под общ. ред. В.С. Авдуевского, В.К. Кошкина. М.: Машиностроение, 1992. 528 с.

[7] Кутателадзе С.С. Основы теории теплообмена. М.: Атомиздат, 1979. $416 \mathrm{c}$.

[8] Колесник С.A. // Мат. моделирование. 2014. Т. 26. № 2. C. $119-132$.

[9] Еремин А.В., Кудинов В.А., Кудинов И.В. // Изв. РАН. Механика жидкости и газа. 2016. Т. 51. № 1. С. 33-44.

[10] Кудинов И.В., Кудинов В.А., Котова Е.В., Еремин А.В. // Инж.-физ. журн. 2017. Т. 90. № 6. С. 1387-1397.

[11] Ненахов Е.В., Карташов Э.М. // Изв. РАН. Энергетика. 2018. № 4. C. 73-86.

[12] Сайбутдинов А.И., Тимеркаев Б.А., Ибрагимов А.Р. // Письма в ЖТФ. 2018. Т. 44. В. 4. С. 74-79.

[13] Formalev V.F., Kolesnik S.A., Kuznetsova E.L. // Composites: Mechanics, Computations, Applications. Int. J. 2018. V. 9. N 3. P. 223-237.

[14] Formalev V.F., Kolesnik S.A. // Int. J. Heat Mass Transfer. 2018. V. 123. P. 994-998.

[15] Формалев В.Ф., Колесник С.А. // Инж.-физ. журн. 2019. T. 92. № 1. C. 55-63. 